Researchers can build relationships with news agencies and lawmakers to translate the science and build trust. Additionally, targeting messaging to rural lawmakers and constituents is critical to build support for evidence-based laws.

\title{
Health disparities and violence
}

\section{SHARED RISK AND PROTECTIVE FACTORS FOR CRIME AND POOR HEALTH ON THE STREET}

${ }^{1}$ Beidi Dong, ${ }^{2}$ Clair White, 'David Weisburd. 'George Mason University; ${ }^{2}$ University of Wyoming

10.1136/injuryprev-2020-savir.74

Statement of Purpose Geographic areas with high rates of crime and violence are often the same places that evidence concentrated, chronic diseases and high morbidity and mortality rates. Yet, little is known about whether and how strategies that target crime and violence can also enhance health outcomes. We aimed to identify the shared risk and protective factors for crime and ill health on the street.

Methods We used survey data from 3,738 respondents living on 449 street segments and physical observation data in Baltimore, Maryland. Mixed effects models were used to partition the variabilities of crime (i.e., general offending, violent offending, and violent victimization) and health outcomes (i.e., general perception of personal health, health limitations, and diagnoses of diseases) at the street versus community level and to identify the shared risk and protective factors on the street while controlling for sociodemographic characteristics.

Results Between $3.8 \%$ and $8.3 \%$ of the total variabilities of crime outcomes are at the community level, whereas between $11.4 \%$ and $12.0 \%$ of the total variabilities of health outcomes are at the community level. Results from regression analyses (i.e., mixed effects model for each of the aforementioned outcomes) indicated that collective efficacy and police legitimacy are two of the most important shared factors when mitigating the undesirable co-occurrence of crime and ill health on the street.

Conclusion The variabilities of crime and health outcomes are more at the street-segment than community level. Shared risk and protective factors exist for reducing the co-occurrence of crime and ill health on the street.

Contributions to Injury and Violence Prevention Science: As members of the criminal justice and public health disciplines often work collaboratively with marginalized populations, it is imperative to address the shared risk and protective factors for public safety and health.

\section{Violence research and prevention in healthcare settings}

\section{DO PARENTS AND KIDS AGREE WHEN REPORTING THE CHILD'S POTENTIALLY TRAUMATIC EVENTS AFTER A VIOLENT INJURY?}

Yansy Salmerón, Katherine Feske-Kirby, Rachel K Myers, Stephanie Garcia, Joel A Fein. Children's Hospital of Philadelphia

10.1136/injuryprev-2020-savir.75
Statement of Purpose Ascertaining an accurate and cumulative history of exposure to potentially traumatic events (PTE) can be valuable in identifying violently-injured youth's recovery needs. It is unclear how youth and caregiver report of youth's PTEs relate to each other. We examined agreement between youth and caregiver self-report of 23 different PTEs following the youth's recent violent injury.

Methods As part of a larger survey, 48 youth ages 8-18 and their caregiver independently completed a self-administered Trauma History Questionnaire (THQ) within approximately 20 days following the youth's Emergency Department visit for a violent injury. We compare youth and caregiver reports of youth PTEs and identify PTEs with the greatest and least agreement.

Results Mean youth participant age was $13.8 \pm 2.2$ years; $42 \%$ were female, $96 \%$ reported nonpenetrating injuries. The average number of PTEs was $9 \pm 4$ by youth report and $8 \pm 4$ by caregiver report. For 13 of the PTEs there was $>50 \%$ youthparent discordance, wherein the youth reported the PTE and the caregiver did not. Youth and caregiver agreement exceeded $80 \%$ for three PTEs, the highest of which reported someone physically hurting or threatening the child.

Conclusion Although youth and parents are reporting a similar number of youth PTEs, there are a substantial number of PTEs for which youth report but parents do not and a small number of PTEs where they usually agree.

Significance and Contributions to Injury and Violence Prevention Science Violently injured youth and their parents often disagree about the youth's exposure to PTEs. This could reflect a youth's failure to disclose the PTEs to their parent, or a difference in their respective appraisals of what events are considered potentially traumatic. Further research will explore how these discrepancies may inform mental health treatment of violently injured youth in the peri-traumatic recovery period.

\section{Health disparities and violence}

\section{7 'THE TOLL THAT IT'S TAKEN:' PERSPECTIVES OF BLACK MEN RECOVERING FROM TRAUMATIC INJURY IN PHILADELPHIA}

'Sara Jacoby, ${ }^{1}$ Jessica Webster, ${ }^{2}$ Catalyst Twomey, ${ }^{2}$ Abeselom Gebreyesus. 'School of Nursing, University of Pennsylvania; ${ }^{2}$ Perelman School of Medicine, University of Pennsylvania

\subsection{6/injuryprev-2020-savir.76}

Statement of Purpose For the majority of individuals who will survive trauma, healing and restoration is often a long-term process shaped by everyday socio-ecological exposures. The purpose of this analysis was to describe how Black men in Philadelphia perceived the long-term sequalae of their injuries and the influence of environmental and structural factors on recovery.

Methods 26 individuals, 12-36 months after injury, were rerecruited from a concluded cohort study which followed over 600 seriously injured Black men from admission to a trauma center in Philadelphia to three months after hospitalization. This sub-sample was generally representative of larger cohort by age, mechanism of injury, insurance status, income, and education. Focus group and individual qualitative interviews 